\title{
Automatic Railway Crack Detector System using LoRa Network
}

\author{
Haritha $\mathbf{P}^{1}$, Sharnya $\mathbf{V}^{2}$ \\ PG Scholar, Department of ECE, CMS College of Engineering and Technology, Coimbatore, India ${ }^{1}$ \\ Assistant Professor, Department of ECE, CMS College of Engineering and Technology, Coimbatore, India ${ }^{2}$
}

\begin{abstract}
Safety and reliability are considered in one of the main issues at all transport system, particularly in railway. An automatic railway track crack detector system for Railway has been proposed here which aims in building a robot that can detect and analyze any kind of crack on the railway line and send the coordinates of that faulty line to the concerned authority. The proposed system can be networked with multiple robots and central computer system can control all these robots, so that complete track can be scanned for detecting any crack before each time train passes through track. Multiple such systems can be connected by using LoRa network instead of GSM network. This will eliminate the connectivity and network traffic issues. With the networked robotic system can be connected with the railway signaling system to synchronize the signaling with the crack detection. Synchronization with the signaling system can help automatic control of traffic if any crack is detected.
\end{abstract}

Keywords: LoRa Network, Robot, PIR sensor, ultrasonic sensor

\section{INTRODUCTION}

Over the years many researchers have tried to build up a system for tracking the cracks in rail lines. Krishna used IR technology to track the cracks and send it through the Bluetooth. Though Bluetooth system is not effective for long range communication. Some researchers used IR transmitter and receiver for railways crack inspection and send the data through GSM module. However, sometimes the IR sensor can detect a small crack which might not be that risky for trains and cause an unnecessary concern. Muley et al proposed an op-amp based system where the crack has been identified by the change of output voltage in the op-amp. Though a noise in the railway tracks can change the voltage and give a false alarm. Rizvietal and Delforouzi et al uses a computer vision based railway crack detection system. It's an effective method to inspect the cracks. However, to detect the cracks in the night it needs high functioning cameras, which increase the cost of the system. There are also some other systems exists to solve the crack problem using different sensors, Zigbee technology and LED-LDR system. But most of this system is designed for other countries rather than Bangladesh. Safety and reliability are considered in one of the main issues at all transport system, particularly in railway. There is a view from the experts that the present regulatory framework does not clarify effective deal with railway accidents and derailments, approximately $60 \%$ of a rail accident is due to the derailment, of which 90\% are due to crack problems. In recent years developing country like Bangladesh needs more diversification and innovation in the transportation system. With the increasing number of the population the current safety framework needs to be equipped with safety legislation and more invulnerable. Safe railway track plays a significant role to carry more than sixty thousand people at a time. Therefore, a consistent system is required to detect the cracks in the railway tracks.

\section{MOTIVATION}

Though a noise in the railway tracks can change the voltage and give a false alarm. Rizviet al and Delforouzi et al uses a computer vision-based railway crack detection system. It's an effective method to inspect the cracks. However, to detect the cracks in the night it needs high functioning cameras, which increase the cost of the system. There are also some other systems exists to solve the crack problem using different sensors, Zigbee technology and LED-LDR system. But most of this system is designed for other countries rather than Bangladesh. In this project, for the first time in Bangladesh, a cost-effective autonomous railway crack detector robot is proposed for Bangladesh railway. This device can generate a complete solution for the railway track that starts with crack detection, fault analysis using ultrasonic sensor and that end up with an SMS alert to the concerned authority which consists of the location of the crack. Therefore, making ease the authority to prevent accident san extra communication with the help of a Relay in between the terminals which thereby enhances the signal quality. 


\author{
Vol. 8, Issue 4, April 2019
}

\title{
III. RELATEDWORKS
}

Track-caused derailments are often caused by wide gauge. Proper gauge, the distance between rails, is 56.5 inches (four feet, eight-and-a-half inches) on standard gauge track. As tracks wear from train traffic, the rails can develop a wear pattern that is somewhat uneven. Uneven wear in the tracks can result in periodic oscillations in the truck, called 'truck hunting.' Truck hunting can be a contributing cause of derailments. A rail breaks cleanly, it is relatively easy to detect. A track occupancy light will light up in the signal tower indicating that a track circuit has been interrupted. If there is no train in the section, the signaller must investigate. One possible reason is a clean rail break. For detecting the rail break this way, one has to use signal bonds that are welded or pin brazed on the head of the rail. If one uses signal bonds that are on the web of the rail, one will have a continued track circuit. If a rail is merely cracked or has an internal fissure, the track circuit will not detect it, because a partially-broken rail will continue to conduct electricity. Partial breaks are particularly dangerous because they create the worst kind of weak point in the rail. The rail may then easily break under load--while a train is passing over it at the point of prior fissure.

An automatic railway track crack detector system for Bangladesh Railway has been proposed here which aims a robot that can detect and analyze any kind of crack on the railway line and send the coordinates of that faulty line to the concerned authority. This robot includes two ultrasonic sensors, GPS, GSM modules, and Arduino Mega based crack detection assembly which is cost effective and robust to facilitate better safety standards in railways. As soon as the robot passed through a crack that might cause the derailment of a train, the ultrasonic sensors sense that and generate a signal. Then this signal is fed into the Arduino Mega. At that point, with the assistance of GSM and GPS modules, an alert SMS consist of the geographic coordinate of that damaged track is sent to the nearby railway authority who can easily take necessary steps to resolve the problem before any major accident occurs. This will save several trains in Bangladesh from an unwanted discontinuity from the rail track.

The principle involved in crack detection is the concept of LDR. In the proposed design, the LED will be attached to one side of the rails and the LDR to the opposite side. During normal operation, when there are no cracks, the LED light does not fall on the LDR and hence the LDR resistance is high. Subsequently, when the LED light falls on the LDR, the resistance of the LDR gets reduced and the amount of reduction will be approximately proportional to the intensity of the incident light. As a consequence, when light from the LED deviates from its path due to the presence of a crack or a break, a sudden decrease in the resistance value of the LDR ensues. This change in resistance indicates the presence of a crack or some other similar structural defect in the rails. In order to detect the current location of the device in case of detection of a crack, a GPS receiver whose function is to receive the current latitude and longitude data is used. To communicate the received information, a GSM modem has been utilized. The GSM modem transfers the received Information to the GPRS which then shows the exact location of the faulty rail track in the mobile

\section{PROPOSED SYSTEM}

Safety and reliability are considered in one of the main issues at all transport system, particularly in railway. There is a view from the experts that the present regulatory framework does not clarify effective deal with railway accidents and derailments, approximately $60 \%$ of a rail accident is due to the derailment, of which $90 \%$ are due to crack problems. An automatic railway track crack detector system for Railway has been proposed here which aims in building a robot that can detect and analyze any kind of crack on the railway line and send the coordinates of that faulty line to the concerned authority. This robot includes two ultrasonic sensors, GPS, GSM modules, and Arduino Mega based crack detection assembly which is cost effective and robust to facilitate better safety standards in railways. As soon as the robot passed through a crack that might cause the derailment of a train, the ultrasonic sensors sense that and generate a signal. Then this signal is fed into the Arduino Mega. At that point, with the assistance of GSM and GPS modules, an alert SMS consist of the geographic coordinate of that damaged track is sent to the nearby railway authority who can easily take necessary steps to resolve the problem before any major accident occurs. This will save several trains from an unwanted discontinuity from the rail track. The proposed system can be networked with multiple robots and central computer system can control all these robots, so that complete track can be scanned for detecting any crack before each time train passes through track. Multiple such systems can be connected by using LoRa network instead of GSM network. This will eliminate the connectivity and network traffic issues. With the networked robotic system can be connected with the railway signalling system to synchronize the signalling with the crack detection. Synchronization with the signalling system can help automatic control of traffic if any crack is detected. For testing purpose instead of LoRa network GSM can be used due to lack of tower for LoRa netwok.

\section{A. LoRa NETWORK}

We can use LoRa network instead of GSM network. This will eliminate the connectivity and network traffic issues. With the networked robotic system can be connected with the railway signaling system to synchronize the signaling with the crack detection. Synchronization with the signaling system can help automatic control of traffic if any crack is 
detected. For testing purpose instead of LoRa network GSM can be used due to lack of tower for LoRa network. A Semtech innovation, LoRa technology offers compelling features for IOT applications including long range, low power consumption and secure data transmission. The technology can be utilized by public, private or hybrid networks and provides greater range than cellular networks. LoRa technology can easily plug into existing infrastructure and enables low cost battery operated IOT applications.

$\begin{array}{ll}\text { - } & \text { Features of Lora Network } \\ \text { 1. } & \text { Long range } \\ 2 . & \text { Low power } \\ \text { 3. } & \text { Secure } \\ \text { 4. } & \text { Standardized } \\ 5 . & \text { Geo-location } \\ 6 . & \text { Mobile } \\ 7 . & \text { High capacity } \\ 8 . & \text { Low cost }\end{array}$

V. EXPERIMENTAL MODEL

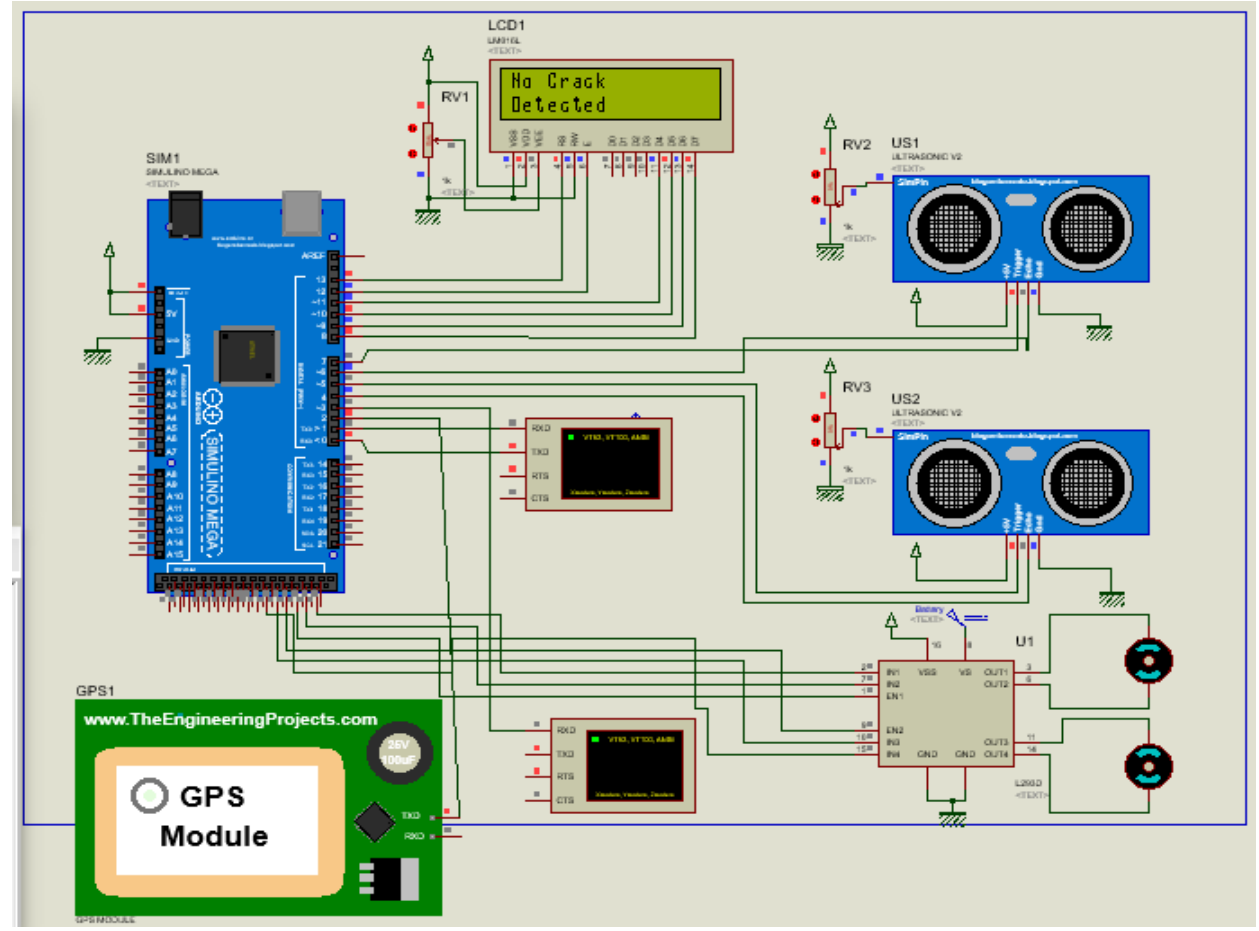

Fig 1: Experimental model of the sytem

This robot includes two ultrasonic sensors, GPS, GSM modules, and Arduino Mega based crack detection assembly which is cost effective and robust to facilitate better safety standards in railways. As soon as the robot passed through a crack that might cause the derailment of a train, the ultrasonic sensors sense that and generate a signal. Then this signal is fed into the Arduino Mega. At that point, with the assistance of GSM and GPS modules, an alert SMS consist of the geographic coordinate of that damaged track is sent to the nearby railway authority who can easily take necessary steps to resolve the problem before any major accident occurs. This will save several trains in Bangladesh from an unwanted discontinuity from the rail track. The proposed system can be networked with multiple robots and central computer system can control all these robots, so that complete track can be scanned for detecting any crack before each time train passes through track. Multiple such systems can be connected by using LoRa network instead of GSM network. This will eliminate the connectivity and network traffic issues.

\section{SIMULATION RESULT}

Proteus simulator potentiometer value was used as alternating of crack for ultrasonic sensors. Therefore, for a specific potentiometer value the ultrasonic sensor sense that as a crack and the real-time results are shown on the LCD display. In the Table I the simulation results are shown. 
IJARCCE

International Journal of Advanced Research in Computer and Communication Engineering

Vol. 8, Issue 4, April 2019

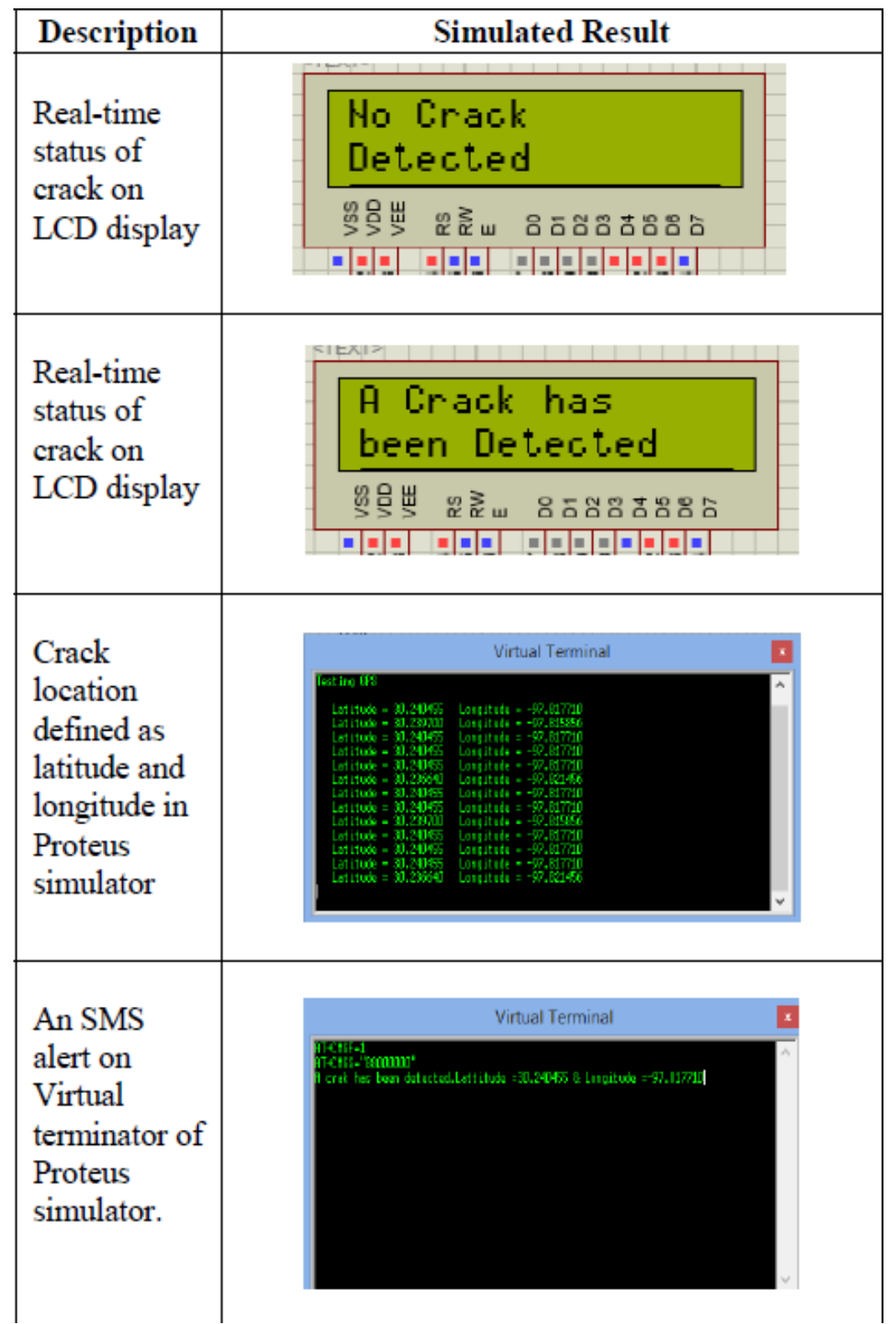

Fig 2: Simulation result

VII. HARDWARE RESULT

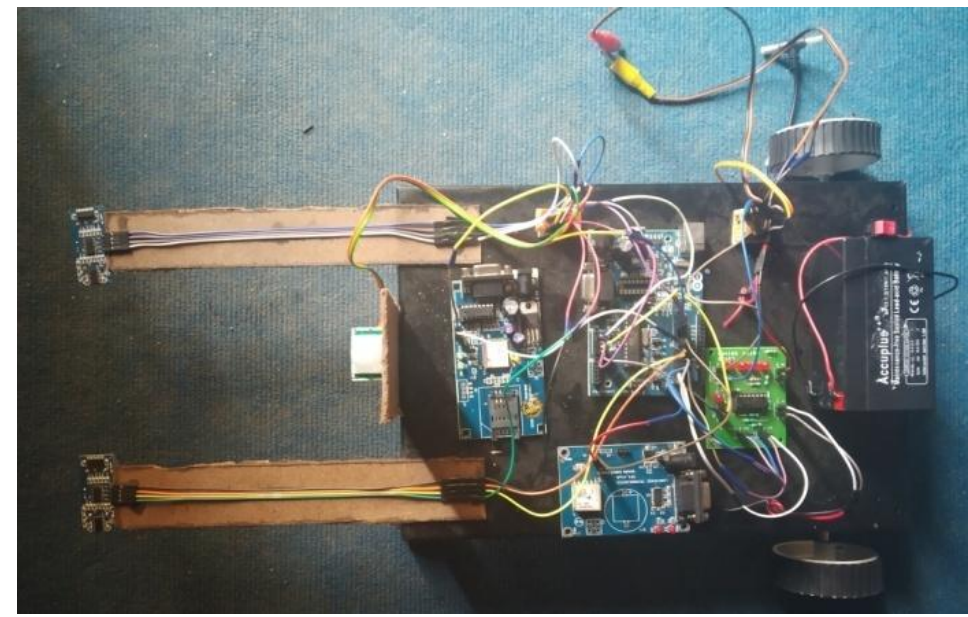

Fig 3 : Hardware result 
Vol. 8, Issue 4, April 2019

The main difference between simulation and hardware analysis is in the simulation we used potentiometers as ultrasonic sensors for sensing crack and virtual terminal to show the location coordinates of the cracks collecting from GPS module and display the alert SMS of GSM modem. If any crack is found on rail lines the robot stops and send the location coordinates to the authority so that they can take essential steps. The robot continues its crack detection if no crack is found.

\section{CONCLUSION}

The main causes of rail accidents are a collision between trains and derailment. Derailment of trains occurs mainly due to cracks in railway tracks. The proposed robust and cost-effective system helps to detect these cracks. In this paper, an autonomous system is designed for railway track security by developing a microcontroller-based robot. This device brings a digital solution for the real-time problem that is a huge threat for thousands of people who are using the train as a major transport in their daily life. The crack can be detected without any error. The government of Bangladesh can take initiative to implement this in large scale. In the long run, it will facilitate better safety standards for rail tracks and provide effective testing infrastructure for achieving better results in the future. This robot will create a revolutionary transformation in the railway security system. For testing purpose instead of LoRa network GSM can be used due to lack of tower for LoRa network.

\section{REFERENCES}

[1]. Ch. MuneedraRao and B.R. BalaJaswanth, "Crack sensing scheme in rail tracking system", Int. Journal of Engineering Research andApplication, Vol. 4, Issue 1, January 2014, pp.13-18.

[2]. B.Siva Rama Krishna, D.V.S Seshendra, G.Govinda Raja, T.Sudharshan, and K.Srikanth, "Railway Track Fault Detection System by Using IR Sensors and Bluetooth Technology", Asian Journal of Applied Science and Technology (AJAST), Volume 1, Issue 6, Pages 82-84, July 2017.

[3]. Rakesh V. Pise, Parag D. Nikhar, Prof. Avinash H. Shelar, "Review on Railway Track Crack Detection Using Ir Transmitter and Receiver"; International Research Journal of Engineering and Technology(IRJET), 4(1), 1723-1726, 2017.

[4]. Lad, P., \& Pawar, M., "Evolution of railway track crack detection system", 2nd IEEE International Symposium on Robotics and Manufacturing Automation, ROMA 2016.

[5]. Er.Nisthul G, Lijo George, Nelson Varghese, Sebin Jose, Nithin John \& Nandhumon K R., "Automatic Railway Track Crack Detection System", Imperial Journal of Interdisciplinary Research (IJIR) Vol-3, Issue-4, 2017.

[6]. Muley, A. S., Patil, S. B., \& Shelar, P. A. H., "Railway track crack detection based on GSM technique", International Research Journal of Engineering and Technology(IRJET), 4(1), 1252-1254, 2017.

[7]. Rizvi Aliza Raza, Khan Pervez Rauf, Ahmad Shafeeq., "Crack Detection in Railway Track Using Image Processing", International Journal of Advance Research, Ideas and Innovations in Technology, Volume-3, Issue-4, 489-496, 2017. 